\title{
THE INVESTIGATION OF TERMINAL SEGMENT EFFECT ON BLOOD PRESSURE PROPAGATION PATTERNS IN CARDIOVASCULAR SYSTEM MODELS
}

The electro-mechanical analogy method is used for the simulation of blood flow in blood vessels. Particularly the subject of this paper is the investigation of the influence of terminal segment topology on the blood pressure propagation patterns. The cardiovascular system is widely branched and therefore very complex. Thus, it is impractical to create the simulation model consisting of all blood vessels and attention is focused to development of models for smaller parts of vascular system. The selection and adjustment of terminal segments for such simulation model is important on behalf of the model accuracy. Three different types of terminal segments were implemented in order to show their influence to the blood pressure and blood flow propagation patterns in two model complexities.

\section{Introduction}

The living organism in general, can be described as a complex system of many component systems. Cardiovascular system is highly complex structure able to regulate itself under physiological condition. Despite this fact some pathological conditions may develop and interfere with self-regulation mechanism (e.g., a fat deposit can reduce vessel section diameter - stenosis, an aneurysm may develop or the arterial wall may become more rigid due to aging). The consequences of these pathologies on the hemodynamical quantities as well as the possible outcome of a treatment may be studied by simulations. Such a model requires patient's data that can be acquired via standard angiologic diagnostic methods (e.g. CT, MRI, angiography, USG).

The principle of duality among hemodynamic characteristic variables and electric variables can be utilised in the process of model development. This approach makes possible to transform physical process from one environment to the other easier solvable one. Despite this, in relation to utilised model type the description of whole circulatory system can become far too complex and simulation model computationally expensive. For detailed description it is usually chosen only small area of interest, which can be exactly separated from the rest of system and its relationship to the other parts can be well defined and described. Comparison of boundary model elements influence to the simulation results for simplified and more complex model of human upper limb is the object of this paper.

\section{Theory}

The blood flow is formally analogical to propagation of electric current along electric lines. Differential equations of hydrodynamic processes in a tube are similar to those describing transmission of electric charge in an electric line.

The blood flow in physiological case is considered to be laminar. As the blood vessels forks, the sum of diameters of all branches can differ from the diameter of the original vessel. This sudden change of tube diameter usually followed with change of tube direction can cause reflections in propagating flow. Reflections or other hydrodynamic processes can occur also in pathologic case, when
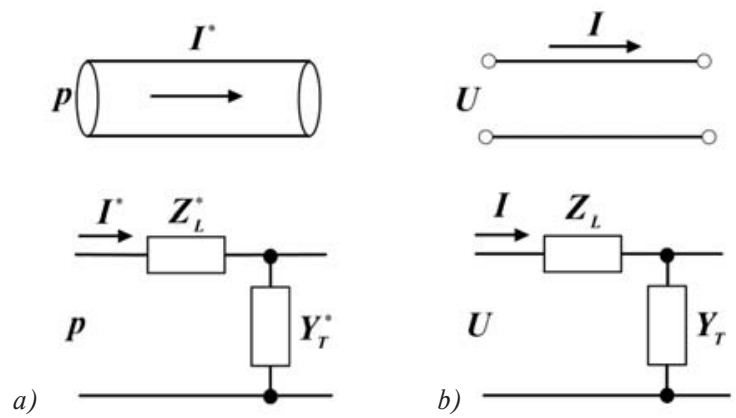

Fig. 1 Analogy of a tube with liquid and an electric line: a) Section of a homogeneous tube, b) Section of a homogeneous two-wire line.

\footnotetext{
* Daniela Gombarska, Barbora Czippelova, Ivo Cap

Faculty of Electrical Engineering of the University of Zilina, Slovakia, E-mail: gombarska@fel.uniza.sk, web: http://fel.uniza.sk/ktebi/
} 
the sudden change in vessel diameter is caused by disease, for example stenosis in vessel wall. [1]

The analogy of hydrodynamic process in a tube and transmission of electric current can be demonstrated by schematic drawing in Fig. 1. Longitudinal impedances $Z_{L}{ }^{*}$ and $Z_{L}$ represent conservative and dissipative components, transversal admittances $Y_{T}{ }^{*}$ and $Y_{T}$ describe cross elasticity and inter-wire capacity and cross losses. The detailed depiction of equivalent circuit for one blood vessel segment is shown in Fig. 2 (a) and is in more detail described in [2]. Values of longitudinal impedance parameters depend on blood flow and blood pressure ratio. Formulas for estimation of elements in longitudinal and transversal part of equivalent circuit are based on work of Gaelings [3]. Elements of equivalent circuit of blood vessel can be derived by means of equations describing hemodynamics (Navier - Stokes equation, continuity equation) and by using electromechanical analogy.

$$
\begin{aligned}
& R_{n}=\frac{8 \eta}{\pi r_{0}^{4}} n, \quad L_{n}=\frac{1}{2 n-1} \frac{\rho}{\pi r_{0}^{2}}, \\
& C_{p}=\frac{2 \pi r_{0}}{\kappa E}, \quad R_{p}=\frac{\kappa \eta_{w}}{2 \pi r_{0}}, n=1,2,3
\end{aligned}
$$

where $r_{0}$ is radius of the vessel wall, $\rho$ is the blood density, $\eta$ dynamic viscosity, $E$ is the volume stiffness of the tube wall and $\eta_{w}$ - coefficient of internal friction (cause of deformation losses), $\kappa$ - geometrical factor. All the longitudinal and transversal parameters of the equivalent circuit are per-unit values.

The cardiovascular system can be divided into cascade sequence of small segments. The model of cardiovascular system is then composed of equivalent circuits of these segments linked together $[3,4,5]$. Despite the electric circuit analogy computation efficiency, modelling of each individual vessel of blood circulatory system is impractical. Therefore, the detailed model is created only for the area of interest and all the contiguous and distal parts are lumped together and substituted by equivalent circuit, Fig. 2b, c and d.

For comparison of terminal segment influence on blood circulation simulation results three types of lumped equivalent circuits are utilised. The simplest model of terminal segment, named Case A, is two-element Windkessel model, shown in Fig. 2b. Parame- ters $R_{T}, C_{T}$ of equivalent circuit represent peripheral resistance and compliance. One possible estimation method of these parameters is by impedance matching. In context of the electrical theory analogy these parameters are derived from characteristic impedance of vessel equivalent circuit. We assume the output impedance of vessel model to match the input impedance of terminal segment. The characteristic impedance of equivalent circuit is

$$
\dot{Z}_{0}=\sqrt{\frac{\dot{Z}_{L}}{\dot{Y}_{T}}},
$$

where $\dot{Z}_{L}$ is longitudinal impedance and $\dot{Y}_{T}$ is transversal admittance of vessel segment equivalent circuit. The terminal segment impedance is

$$
\dot{Z}_{T}=\frac{R_{T}\left(-j X_{C_{T}}\right)}{R_{T}-j X_{C_{T}}},
$$

The reflection in pulse and blood flow wave depends according to electric transmission line theory on the difference between the impedance of the line at the joint and impedance at the terminal segment.

$$
\dot{r}=\frac{P^{-}}{P^{+}}=\frac{\dot{Z}_{T}-\dot{Z}_{0}}{\dot{Z}_{T}+\dot{Z}_{0}},
$$

Because in general within the periphery blood circulatory system is supposed to operate without reflections the reflection coefficient $\dot{r}=0$ and $\dot{Z}_{T}=\dot{Z}_{0}$. Thus the $R_{T}, C_{T}$ parameters of equivalent circuit are derived combining equations (3) and (4), from real and imaginary parts of $\dot{Z}_{0}$.

Second type of terminal segment topology, Case B in Fig. 2 (c), is three-element Windkessel model. Calculation of the parameters $R_{1}, R_{2}$ and $C_{2}$ is also derived from electric transmission lines theory - vessel segment characteristic impedance and terminal segment impedance [8].

$$
\dot{Z}_{T}=R_{1}+\frac{R_{2}\left(-j X_{C 2}\right)}{R_{2}-j X_{C_{2}}},
$$

Total terminal resistance $R_{T}$ is in this type of terminal segment equal to sum of $R_{1}$ and $R_{2}$. For derivation of $R_{1}, R_{2}$ and $C_{2}$ are applied the same assumptions as in two-element Windkessel model. However in this case the derivation leads to quadratic equation and only physically meaningful results should be taken into account.

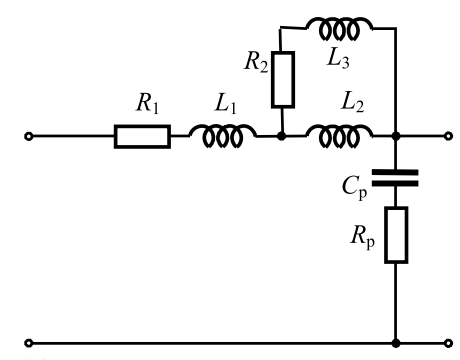

(a)

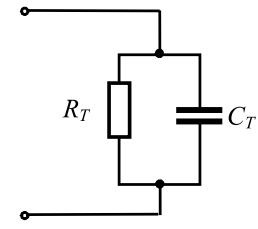

(b)

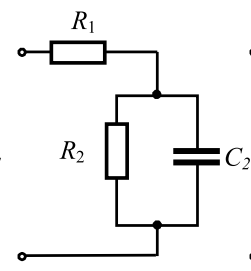

(c)

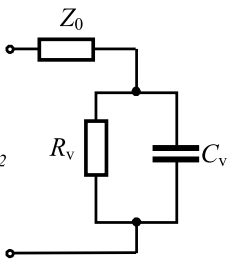

(d)

Fig. 2 a) Equivalent circuit of one segment of distributed parameters model, b) Case A, c) Case B and d) Case $C$ are equivalent circuits of terminal segment 
Case C in Fig. 2d is topologically similar to the Case B. However, for the parameter estimation the different approach is chosen. In previous two cases the transmission line is supposed to be lossy. Here we assume that the cut-off line is infinitely long and lossless [9]. Thus the characteristic impedance is frequency independent and depends only on the longitudinal inertia per segment length $\mathrm{L}$ and the compliance per segment length $\mathrm{C}$

$$
Z_{0}=\sqrt{\frac{L}{C}} .
$$

The characteristic impedance $\dot{Z}_{0}$ and resistance of distal segments $R_{v}$ compose the total terminal resistance $R_{T}=Z_{0}+R_{v}$. Compliance of the distal part behind the end segment $C_{v}$ can be calculated with time constant $\tau$

$$
C_{v}=\frac{\tau}{R_{v}}
$$

According to [9] the typical value for $\tau$ is $1.5 \mathrm{~s}$.

In all three terminal segment models is total terminal resistance expressed by means of mean pressure and mean flow with $\alpha$ the percentage of the cardiac output flowing through the vascular bed behind the end segment [9]

$$
R_{T}=\frac{p}{\alpha q} .
$$

\section{Simulation and Results}

For examination of terminal segment effect we chose human arm arteries, Fig. 3.

From the complexity point of view, two model structures are simulated. The first one, more complex model of human upper limb arterial tree consists of 24 segments of arteries (Brachialis, Radialis,
Ulnaris and Arcus Palmaris) and 8 terminal segments for outgoing arteries (Profunda Brachii, Superior Ulnar Collateral, Inferior Ulnar Collateral, Interosseus, Policis and three Digitalis). The simplified version of the model consists of 19 segments of arteries (Brachialis, Radialis and Ulnaris) and 6 terminal segments for outgoing arteries (Profunda Brachii, Superior Ulnar Collateral, Inferior Ulnar Collateral, Interosseus, two parts of Arcus Palmaris).

Pressure waves are computed at two parts of model - at A. Radialis and A. Digitalis. The computation at A. Radialis is carried out at four points - on input of segments 16,17,18 and 19 for both the complex and simplified models. In more complex model the additional computation point is joined at A. Digitalis for comparison with sample of clinical measured data. The results of simulations are compared together to evaluate the influence of terminal segment type on pressure wave form.

Fig. 3c shows typical waveform and amplitude of pulse wave on A. Radialis. The results of simulation for both the model complexities and all three types of terminal segments are displayed in Fig. 5a, b and c.

The influence of terminal segment on the waveform match between the complex model and the simplified one is apparent from Fig. 5a, b and c. In accordance with transmission lines theory the blood pressure wave depends not only on time but also on the distance on the vessel, Figs. 6, 7 and 8. The amplitude of blood pressure increases with distance from the beginning of the vessel. This effect originates from circulatory system geometry, especially in branching parts. Similarly with the transmission line theory, there are segments there, terminated with impedance different from its characteristic impedance. Also the difference between pressure results for more complex and simplified model depends on the distance $x$ on the vessel. The computed mean value of blood pressure difference $\Delta \varepsilon$ between complex and simplified model is shown

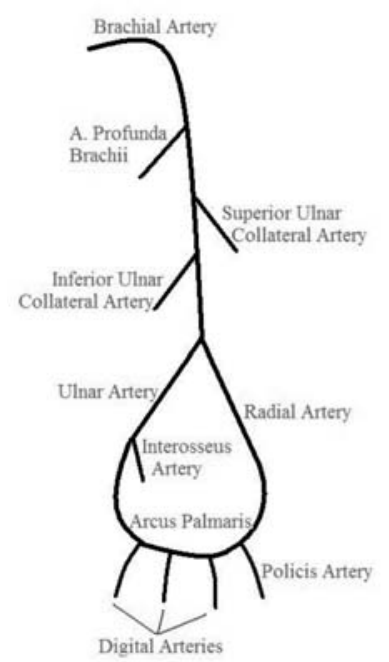

(a)

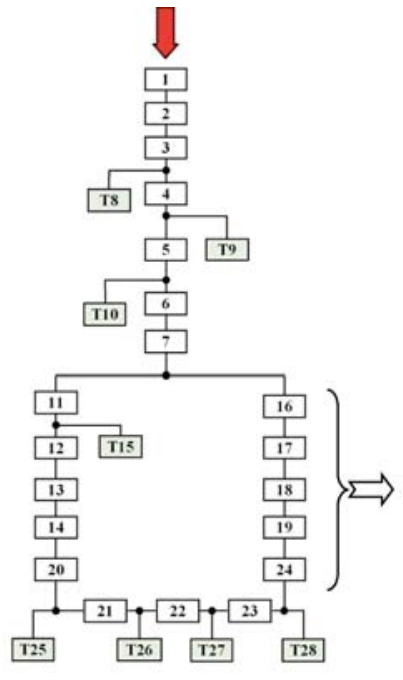

(b)

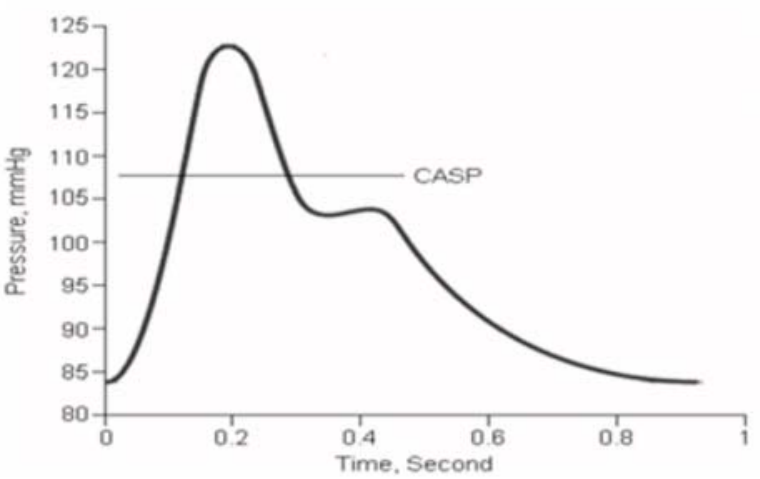

c)

Fig. 3 a) scheme of arm arteries, b) model of arm composed of equivalent circuit segments, c) pressure waveform example in A. Radialis 


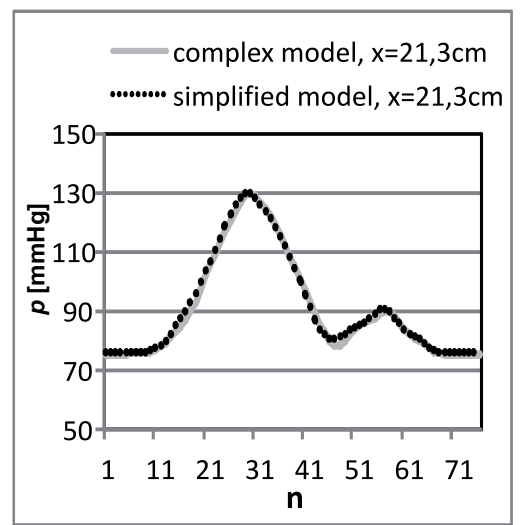

a)

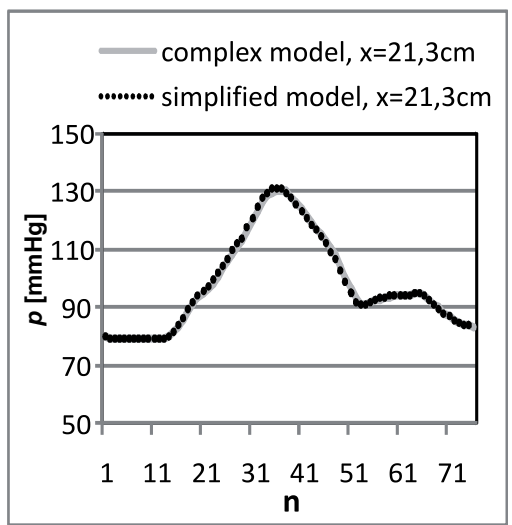

b)

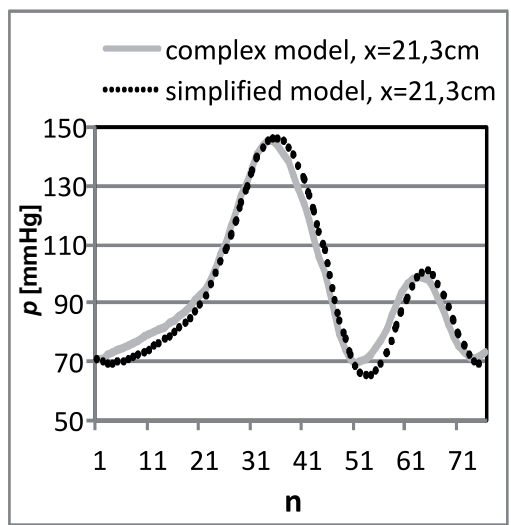

c)

Fig. 5 Pressure waveform in A. Radialis in $x=21,3 \mathrm{~cm}$ for: a) Case A terminal segment,

b) Case B terminal segment and c) Case $C$ terminal segment
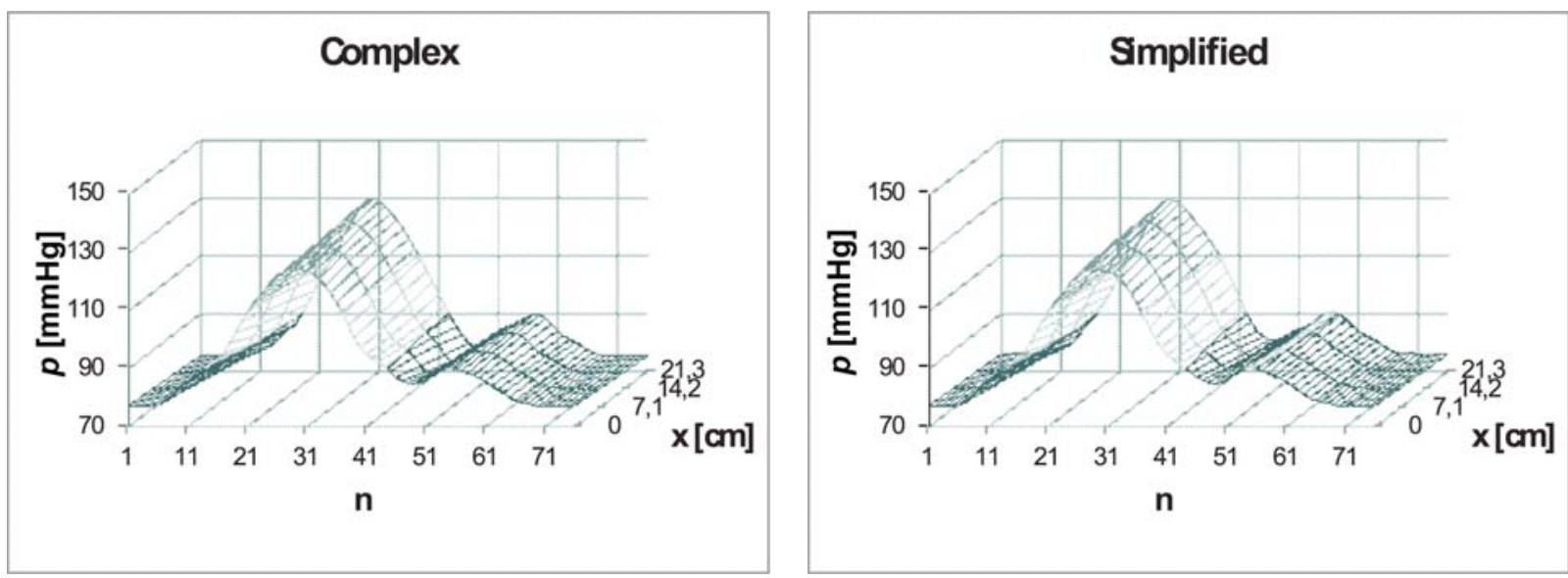

Fig. 6 Calculated pressure waveform vs. sample and distance $x$ in A. Radialis for case A, complex and simplified model
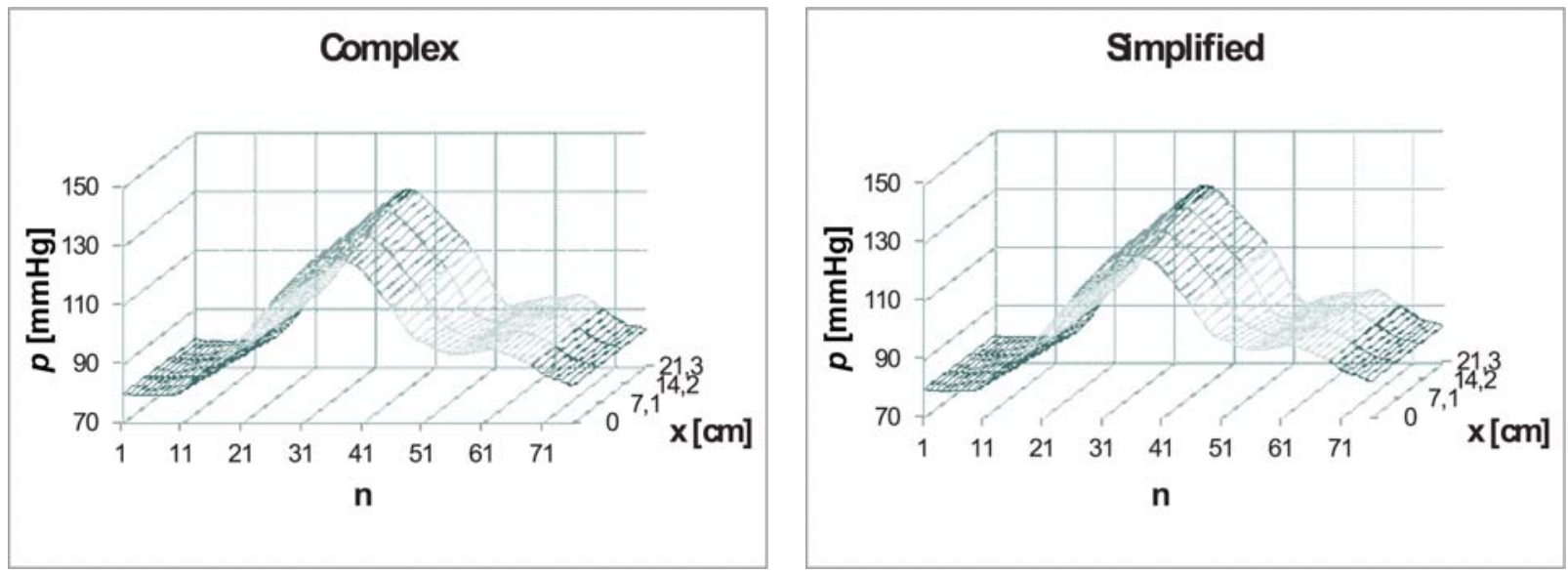

Fig. 7 Calculated pressure waveform vs. sample and distance $x$ in A. Radialis for case B, complex and simplified model 

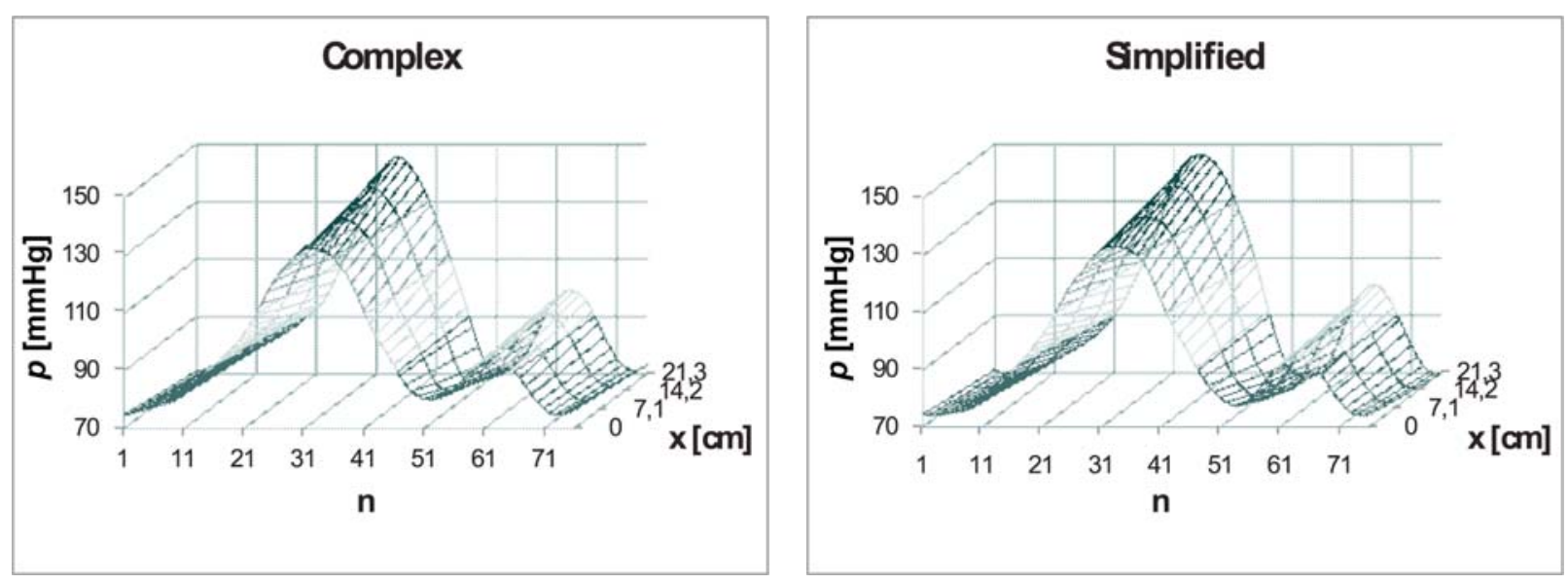

Fig. 8 Calculated pressure waveform vs. sample and distance $x$ in A. Radialis for case C, complex and simplified model

in Fig. 9. The Case B terminal segment appears to have best match between simplified and complex model.

For illustration of the model nature the pressure in A. Digitalis for complex model is computed, Fig. 10a and the measured pressure wave example is shown in Fig. 10b. It is apparent that for the comparison with physiological values the model should be adjusted to the measured subject parameters. However, the pressure waveform of Case A and B show a good affinity to the measured one.

\section{Conclusion}

To show the difference between the three models of terminal segments Case A, Case B and Case C we used the two models of arm arterial tree. First one was the more complex one and the second was simplified by cutting the lower part of arteries off and substituting them by the same type of terminal segments as in a more complex model.

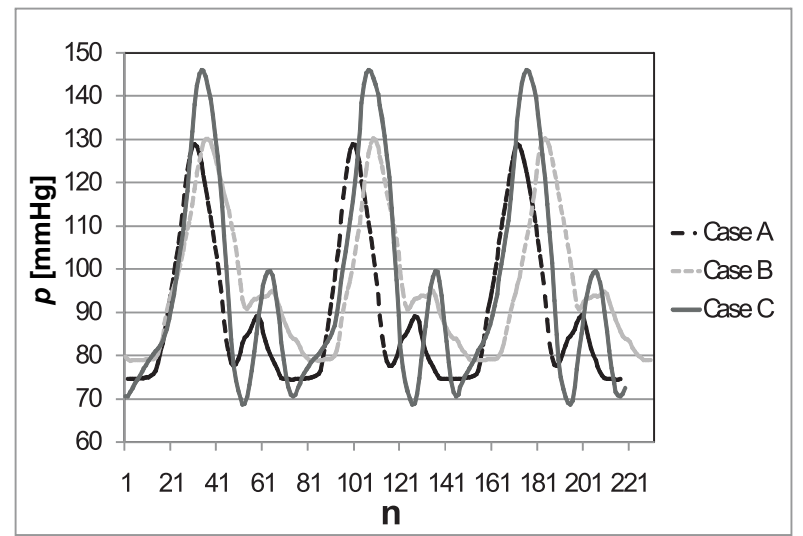

a)

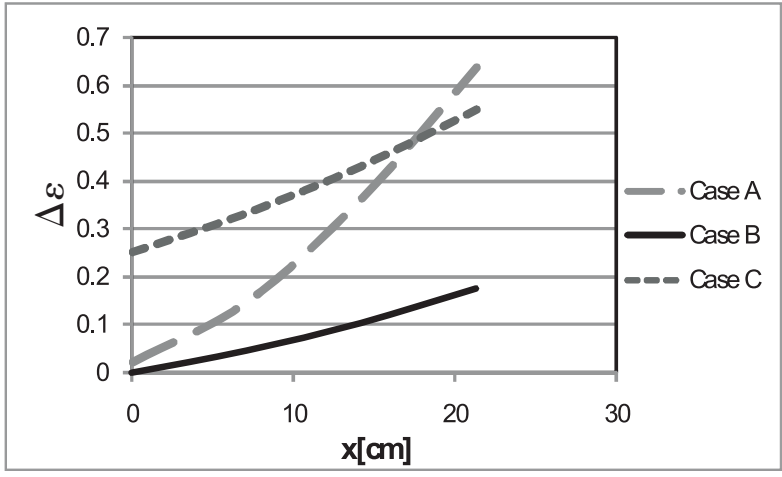

Fig. 9 Mean $\Delta \varepsilon$ vs. distance $x$

From all the simulated cases the best matching showed the Case B - three element Windkessel model. The Case A - two element Windkessel model had similar influence on the pressure wave on the beginning of the vessel, but the difference increases

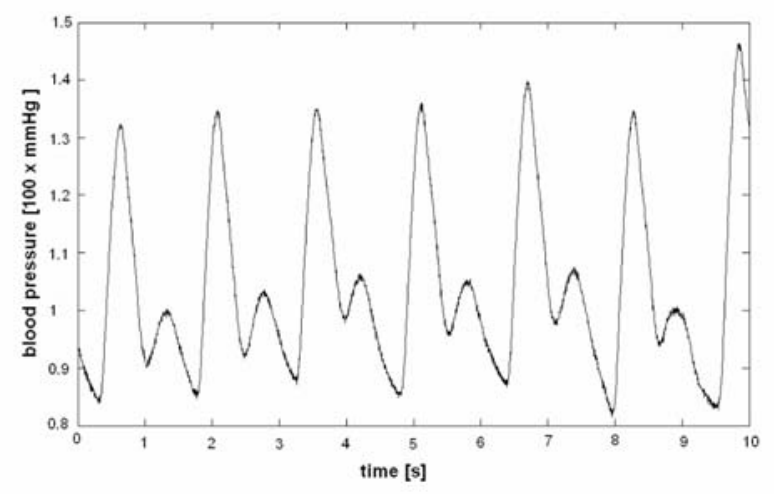

b)

Fig. 10 a) Calculated pressure waveforms in A. Digitalis; b) Measured pressure waveforms in A. Digitalis 
with the distance. The third model of terminal segment Case C appears to be unsuitable for arterial circulatory system simulation purposes.

The human vascular system is highly complex structure with self-regulation ability. It should be emphasized that for the com- parison with physiological values the model parameters should be adjusted for the measured subject. Also some regulatory mechanisms of the system should be considered in the model. All parameters for simulated arteries were obtained from the literature [11], [12]. Therefore the results of this study should be considered as strictly theoretical.

\section{References}

[1] CZIPPELOVA, B., GOMBARSKA, D.: Investigation of Blood Pressure and Blood Flow Wave Propagation on Arterial Bifurcation, In IEEE 2009 - Kraliky, Proc. of conference, Vysoke uceni technicke v Brne, Fakulta elektrotechniky a komunikacnich technologii, 2009. ISBN 978-80-214-3938-2, p. 44-47

[2] CAP, I., CZIPPELOVA, B.: Electromechanical Model of Blood Flow in Vessels, Advances in Electrical and Electronic Engineering, Vol. 7, 2008, pp. 338-341, ISSN 1336 - 1376

[3] GAELINGS, E. W.: Numerische Simulation Haemodynamischer Processe in Vascularen Netzen, Shaker Verlag Aachen, 1996, ISBN3-8265-1509-9, Germany.

[4] WESTERHOF N., STERGIOPULOS N, NOBLE, M.I.M.: Snapshots of Hemodynamics, ISBN 0-387-23345-8

[5] WANG, J.J., PARKER, K.H.: Wave Propagation in a Model of the Arterial Circulation, J. of Biomechanics, Vol. 37, No. 4, 2004, pp. 457-470, ISSN 0021-9290

[6] CZIPPELOVA, B., CAP, I.: Pulse Wave Velocity Calculation by Means of Electromechanical Model of Blood Vessel, Trends in Biomedical Engineering, 2009, pp 149-153, ISBN 978-80-227-3105-8

[7] SACKL-PIETSCH, E.: Continuous Non-invasive Arterial Pressure Shows High Accuracy in Comparison to Invasive Intra-arterial Blood Pressure Measurement, online https://www.biopac.com/Manuals/nibp100d_white_paper.pdf [1.4.2010]

[8] WAITE, L, FINNE, J.: Applied Biofluid Mechanics, The McGraw.Hill companies, United States of America, 2007, ISBN-13: 9780-07-147217-3; ISBN-13:0-07-147217-7

[9] EMMER, M., Lumped Parameter and Experimental Model of Endoleakage After Endovascular Treatment of Abdominal Aortic Aneurysms, Master thesis, Eindhoven University of Technology, 2004

[10] STERGIOPUlOS, N., SEGERS, P., WESTERHOF, N.: Use of Pulse Pressure Method for Estimating Total Arterial Compliance in Vivo, Am J Physiol Heart Circ Physiol, Vol. 276, No. 2, pp 424-428, 1999, ISSN: 0363-6135

[11] JOHN, L. R.: Forward Electrical Transmission Line Model of the Human Arterial System, Medical \& Biological Engineering \& Computing, Vol. 42, pp 312-321, 2004, ISSN: 1741-0444 (Online)

[12] WANG, J. J., PARKER, K. H.: Wave Propagation in Model of the Arterial Circulation, J. of Biomechanics, Vol. 37, No. 4, pp. 425-593, 2004, ISSN: 0021-9290. 